DESY 11-194

\title{
Higgs-induced lepton flavor violation
}

\author{
Andreas Goudelis ${ }^{1}$, Oleg Lebedev ${ }^{1}$ and Jae-hyeon Park ${ }^{2}$ \\ ${ }^{1}$ DESY Theory Group, Notkestraße 85, D-22607 Hamburg, Germany \\ ${ }^{2}$ Institut für Kern- und Teilchenphysik, TU Dresden, 01069 Dresden, Germany
}

\begin{abstract}
Due to the smallness of the lepton Yukawa couplings, higher-dimensional operators can give a significant contribution to the lepton masses. In this case, the lepton mass matrix and the matrix of lepton-Higgs couplings are misaligned leading to lepton flavor violation (LFV) mediated by the Standard Model Higgs boson. We derive model-independent bounds on the Higgs flavor violating couplings and quantify LFV in decays of leptons and electric dipole moments for a class of lepton-Higgs operators contributing to lepton masses. We find significant Higgs-mediated LFV effects at both 1-loop and 2-loop levels.
\end{abstract}




\section{Introduction}

The flavor puzzle of the Standard Model remains one of the outstanding problems in particle physics. Masses of elementary fermions range over many orders of magnitude, forming a pattern which cannot be explained within the Standard Model. Also, it is not known whether these masses are generated by renormalizable terms in the Lagrangian or higher order operators also contribute [1, 2]. This issue will be studied at the LHC by means of the Higgs couplings measurements [3], which will give us at least partial answers. Complementary information about possible effects of non-renormalizable operators is provided by observables sensitive to flavor violation.

In this work, we focus on the lepton sector due to its particular sensitivity to flavor violation. We will consider a class of higher dimensional operators involving the Higgs field which affect the lepton masses. Although such operators are suppressed by a "new physics" scale $M$, they can still give a significant contribution to the lepton masses due to the smallness of the lepton Yukawa couplings. In particular, we will focus on operators of the type

$$
-\Delta \mathcal{L}=Y_{i j}^{(0)} H \bar{L}_{L i} l_{R j}+Y_{i j}^{(1)} \frac{H^{\dagger} H}{M^{2}} H \bar{L}_{L i} l_{R j}+\ldots+\text { h.c. },
$$

as well as higher order operators. Here $Y_{i j}^{(0)}$ and $Y_{i j}^{(1)}$ are a priori independent flavor matrices. Since the resulting lepton mass matrix and the matrix of the Higgs couplings are in general misaligned in flavor space, the presence of higher order operators leads to flavor violation mediated by the Standard Model Higgs boson. The rotation to the mass eigenstate basis may involve large angles, as hinted by the neutrino sector, which amplifies the effect. For example, the processes involving light generations such as $\mu \rightarrow e \gamma$ can be dominated by loops involving the $\tau$ lepton. The existing bounds on LFV observables then place strict constraints on the scale of new physics and/or the type of admissible Yukawa textures.

Some aspects of lepton flavor violation in Higgs interactions have been considered before (for early studies, see [4, [5, 6]), although a systematic study is lacking. For example, LFV Higgs decays $h \rightarrow l_{i} l_{j}$ were analyzed in [7, 8]. The Higgs-mediated decay $\tau \rightarrow \mu \gamma$ was considered in [9], where it was found that this mode is not particularly constraining. Effects of higher order $H^{\dagger} H$-operators on neutrino masses and LFV in a 2 Higgs doublet model were studied in [10]. Finally, Ref. [11] summarizes Higgs-induced quark FCNC effects in extended Higgs models.

The paper is organized as follows. In Section 2, we derive model-independent bounds on the flavor violating couplings of the Higgs boson. In Section 3.1, we apply these bounds to the case 
of dimension 6 operators and obtain constraints on the scale of new physics and on the rotation angles. In Section 3.2, we study the possibility that the lepton mass hierarchy is created entirely by non-renormalizable operators. In Section 4, we present our conclusions.

\section{Bounds on the Higgs couplings}

The relevant Lagrangian describing interactions of the physical Higgs boson $h$ with leptons is given by

$$
\Delta \mathcal{L}=-\frac{y_{i j}}{\sqrt{2}} h \bar{l}_{i} P_{R} l_{j}+\text { h.c. }
$$

where $P_{R}=\left(1+\gamma_{5}\right) / 2$. This interaction induces the following flavor changing dipole operator (Fig. 1),

$$
\mathcal{L}_{\text {eff }_{1}}=e L_{i j} \bar{l}_{i} \sigma^{\mu \nu} F_{\mu \nu} P_{L} l_{j}+\text { h.c. }
$$

where $\sigma^{\mu \nu}=\frac{i}{2}\left[\gamma^{\mu}, \gamma^{\nu}\right], P_{L}=\left(1-\gamma_{5}\right) / 2$, and

$$
L_{i j}=\frac{y_{3 i}^{*} y_{j 3}^{*}}{64 \pi^{2} m_{h}^{2}} m_{\tau} \ln \frac{m_{\tau}^{2}}{m_{h}^{2}} .
$$

Here we have kept only the leading in $m_{\tau} / m_{h} \tau$-lepton contribution in the loop since the others are negligible for our purposes. The corresponding branching ratio is given by

$$
\mathrm{BR}\left(l_{j} \rightarrow l_{i} \gamma\right)=\mathrm{BR}\left(l_{j} \rightarrow l_{i} \nu_{j} \bar{\nu}_{i}\right) \times \frac{192 \pi^{3} \alpha}{G_{F}^{2} m_{j}^{2}}\left(\left|L_{i j}\right|^{2}+\left|L_{j i}\right|^{2}\right) .
$$

The Higgs interactions also induce flavor-diagonal dipole operators at one loop:

$$
\mathcal{L}_{\text {eff }_{2}}=e \operatorname{Re} L_{i i} \bar{l}_{i} \sigma^{\mu \nu} F_{\mu \nu} l_{i}-i e \operatorname{Im} L_{i i} \bar{l}_{i} \sigma^{\mu \nu} F_{\mu \nu} \gamma_{5} l_{i}+\text { h.c. }
$$

These are constrained by the charged lepton anomalous magnetic moments and electric dipole moments,

$$
\begin{aligned}
\left|\delta a_{i}\right| & =4 m_{i}\left|\operatorname{Re} L_{i i}\right| \\
\left|d_{i}\right| & =2 e\left|\operatorname{Im} L_{i i}\right|
\end{aligned}
$$

Finally, there are tree-level processes $l_{j} \rightarrow l_{i} l_{k} l_{k}^{+}$induced by (2). Their branching ratio is given by

$$
\operatorname{BR}\left(l_{j} \rightarrow l_{i} l_{k} l_{k}^{+}\right)=\operatorname{BR}\left(l_{j} \rightarrow l_{i} \nu_{j} \bar{\nu}_{i}\right) \times \frac{\left(4-\delta_{i k}\right)}{256 G_{F}^{2} m_{h}^{4}}\left|y_{k k}\right|^{2}\left(\left|y_{i j}\right|^{2}+\left|y_{j i}\right|^{2}\right)
$$

The resulting bounds on the Higgs couplings are presented in Table 1. Most experimental constraints are taken from Particle Data Group [12, while the recent bound on $\operatorname{BR}(\mu \rightarrow e \gamma)$ is 


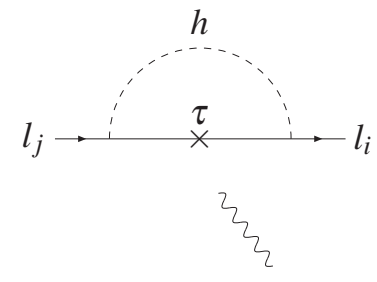

(a)

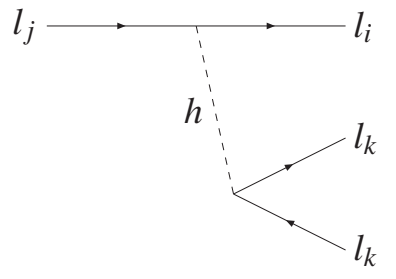

(b)

Figure 1: Leading Higgs-mediated contributions to the lepton dipole operators and 3-body decays.

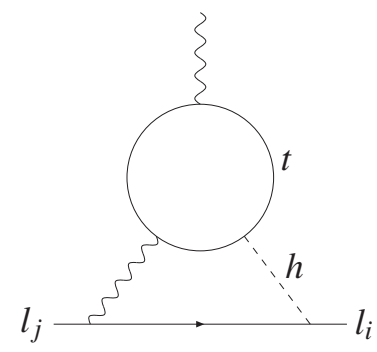

Figure 2: Leading 2-loop Higgs-mediated contribution to the lepton dipole operators.

from [13] and the interpretation of $\delta a_{e}$ is due to [14]. The third column shows the combination of Higgs couplings constrained by a particular observable, while the fourth one shows representative bounds on the couplings under the assumptions $y_{i j}=y_{j i}$ and $y_{i i}=m_{i} / v$, as in the Standard Model 1.1

\section{$2.1 \quad 2-$-loop contributions}

Two loop diagrams of certain types can be important as they are suppressed by only one power of small Yukawa couplings [4]. In what follows, we include the leading Barr-Zee type diagrams [15] with the top quark in the loop (Fig. 2] [16. Since the coupling of the top quark to the Higgs is essentially unaffected by the higher dimensional operators, we will assume the SM value $y_{t t}=1$. Then [16],

$$
\mathrm{BR}\left(l_{j} \rightarrow l_{i} \gamma\right)=\mathrm{BR}\left(l_{j} \rightarrow l_{i} \nu_{j} \bar{\nu}_{i}\right) \times \frac{8 \alpha^{3} v^{2}}{3 \pi^{3} m_{j}^{2}} f^{2}(z)\left(\left|y_{i j}\right|^{2}+\left|y_{j i}\right|^{2}\right),
$$

where $v=174 \mathrm{GeV}$ and

$$
f(z)=\frac{1}{2} z \int_{0}^{1} d x \frac{1-2 x(1-x)}{x(1-x)-z} \ln \frac{x(1-x)}{z},
$$

\footnotetext{
${ }^{1}$ Here we have not included the bound from $\mu$ to $e$ conversion on nuclei since it depends on the Higgs couplings to light quarks, which are uncertain in our framework. In any case, for the SM Higgs-quark couplings, the bound is of order $\sqrt{\left|y_{12}\right|^{2}+\left|y_{21}\right|^{2}}<5 \times 10^{-4}$ for $m_{h}=200 \mathrm{GeV}$, which is superceded by the 2-loop Barr-Zee bound to be studied below.
} 


\begin{tabular}{|l|c|c|l|}
\hline observable & present limit & constraint & $\begin{array}{l}\text { constraint for } \\
y_{i j}=y_{j i}, y_{i i}=m_{i} / v\end{array}$ \\
\hline $\operatorname{BR}(\mu \rightarrow e \gamma)$ & $2.4 \times 10^{-12}$ & $\left(\left|y_{31} y_{23}\right|^{2}+\left|y_{32} y_{13}\right|^{2}\right)^{1 / 4}<7 \times 10^{-4}$ & $\sqrt{\left|y_{13} y_{23}\right|}<6 \times 10^{-4}$ \\
$\operatorname{BR}(\tau \rightarrow \mu \gamma)$ & $4.4 \times 10^{-8}$ & $\left(\left|y_{33}\right|^{2}\left(\left|y_{32}\right|^{2}+\left|y_{23}\right|^{2}\right)\right)^{1 / 4}<5 \times 10^{-2}$ & $\left|y_{23}\right|<2 \times 10^{-1}$ \\
$\operatorname{BR}(\tau \rightarrow e \gamma)$ & $3.3 \times 10^{-8}$ & $\left(\left|y_{33}\right|^{2}\left(\left|y_{31}\right|^{2}+\left|y_{13}\right|^{2}\right)\right)^{1 / 4}<5 \times 10^{-2}$ & $\left|y_{13}\right|<2 \times 10^{-1}$ \\
$\operatorname{BR}(\mu \rightarrow e e e)$ & $1.0 \times 10^{-12}$ & $\left(\left|y_{11}\right|^{2}\left(\left|y_{21}\right|^{2}+\left|y_{12}\right|^{2}\right)\right)^{1 / 4}<2 \times 10^{-3}$ & $\left|y_{12}\right|<1$ \\
$\operatorname{BR}(\tau \rightarrow \mu \mu \mu)$ & $2.1 \times 10^{-8}$ & $\left(\left|y_{22}\right|^{2}\left(\left|y_{23}\right|^{2}+\left|y_{32}\right|^{2}\right)\right)^{1 / 4}<4 \times 10^{-2}$ & $\left|y_{23}\right|<1.7$ \\
$\operatorname{BR}(\tau \rightarrow e e e)$ & $2.7 \times 10^{-8}$ & $\left(\left|y_{11}\right|^{2}\left(\left|y_{13}\right|^{2}+\left|y_{31}\right|^{2}\right)\right)^{1 / 4}<4 \times 10^{-2}$ & $\left|y_{13}\right|<\mathcal{O}\left(10^{2}\right)$ \\
$\operatorname{BR}(\tau \rightarrow e \mu \mu)$ & $2.7 \times 10^{-8}$ & $\left(\left|y_{22}\right|^{2}\left(\left|y_{13}\right|^{2}+\left|y_{31}\right|^{2}\right)\right)^{1 / 4}<4 \times 10^{-2}$ & $\left|y_{13}\right|<1.7$ \\
\hline$d_{e}(\mathrm{e} \cdot \mathrm{cm})$ & $1.1 \times 10^{-27}$ & $\sqrt{\left|\operatorname{Im}\left(y_{31} y_{13}\right)\right|}<2 \times 10^{-4}$ & $\sqrt{\left|\operatorname{Im}\left(y_{13}^{2}\right)\right|}<2 \times 10^{-4}$ \\
$d_{\mu}(\mathrm{e} \cdot \mathrm{cm})$ & $3.7 \times 10^{-19}$ & $\sqrt{\left|\operatorname{Im}\left(y_{32} y_{23}\right)\right|}<4.1$ & $\sqrt{\left|\operatorname{Im}\left(y_{23}^{2}\right)\right|}<4.1$ \\
$\delta a_{e}$ & $2.3 \times 10^{-11}$ & $\sqrt{\left|\operatorname{Re}\left(y_{31} y_{13}\right)\right|}<0.14$ & $\sqrt{\left|\operatorname{Re}\left(y_{13}^{2}\right)\right|}<0.14$ \\
$\delta a_{\mu}$ & $40 \times 10^{-10}$ & $\sqrt{\left|\operatorname{Re}\left(y_{32} y_{23}\right)\right|}<0.13$ & $\sqrt{\left|\operatorname{Re}\left(y_{23}^{2}\right)\right|}<0.13$ \\
\hline
\end{tabular}

Table 1: Current experimental limits on flavor and CP violating observables in the lepton sector, and the corresponding constraints on the Higgs couplings. The displayed bounds on $y_{i j}$ correspond to $m_{h}=200 \mathrm{GeV}$; for other Higgs masses they are to be multiplied by $m_{h} /(200$ $\mathrm{GeV})$.

\begin{tabular}{|c|c|}
\hline observable & constraint \\
\hline $\operatorname{BR}(\mu \rightarrow e \gamma)$ & $\left(\left|y_{12}\right|^{2}+\left|y_{21}\right|^{2}\right)^{1 / 2}<6 \times 10^{-6}$ \\
$\operatorname{BR}(\tau \rightarrow \mu \gamma)$ & $\left(\left|y_{23}\right|^{2}+\left|y_{32}\right|^{2}\right)^{1 / 2}<4 \times 10^{-2}$ \\
$\operatorname{BR}(\tau \rightarrow e \gamma)$ & $\left(\left|y_{13}\right|^{2}+\left|y_{31}\right|^{2}\right)^{1 / 2}<3 \times 10^{-2}$ \\
$d_{e}$ & $\left|\operatorname{Im} y_{11}\right|<6 \times 10^{-7}$ \\
$d_{\mu}$ & $\left|\operatorname{Im} y_{22}\right|<\mathcal{O}\left(10^{2}\right)$ \\
$\delta a_{e}$ & $\left|\operatorname{Re} y_{11}\right|<0.27$ \\
$\delta a_{\mu}$ & $\operatorname{Re} y_{22} \mid<0.21$ \\
\hline
\end{tabular}

Table 2: Two loop constraints for $m_{h}=200 \mathrm{GeV}$. (The bounds get tighter by a factor of 1.5 for $m_{h}=100 \mathrm{GeV}$.) 
with $z=m_{t}^{2} / m_{h}^{2}$. The resulting constraints are presented in Table 2. Since $f(z)$ varies from 0.76 for $m_{h}=200 \mathrm{GeV}$ to 1.14 for $m_{h}=100 \mathrm{GeV}$, these bounds are not very sensitive to the Higgs mass.

The flavor diagonal transitions can also be extracted from the amplitude computed in [16]. We find

$$
L_{i i}=\frac{\alpha}{24 v \pi^{3}} f(z) y_{i i}^{*}
$$

The EDMs and $g-2$ then constrain the diagonal couplings as summarized in Table 2. We note that the bounds from $\operatorname{BR}(\mu \rightarrow e \gamma)$ and $d_{e}$ are particularly important ${ }^{2}$

\section{Lepton flavor violation from Yukawa-type interactions}

\subsection{Dimension-6 operators}

The lepton sector is particularly sensitive to BSM flavor structures due to the smallness of the lepton masses and strong constraints on lepton flavor violation. Since the lepton Yukawa couplings in the SM can be as small as $10^{-5}$, higher dimensional operators involving the Higgs field can give a significant contribution to the lepton masses. In this section we consider an effect of dimension 6 operators of this sort,

$$
-\Delta \mathcal{L}=H \bar{L}_{L i} l_{R j}\left(Y_{i j}^{(0)}+Y_{i j}^{(1)} \frac{H^{\dagger} H}{M^{2}}\right)+\text { h.c. },
$$

which amounts to replacing the constant SM Yukawa couplings with Higgs-dependent ones,

$$
Y_{i j}=Y_{i j}^{(0)}+Y_{i j}^{(1)} \frac{H^{\dagger} H}{M^{2}}
$$

Here $Y_{i j}^{(0)}, Y_{i j}^{(1)}$ are in general independent flavor matrices and $M$ is the new physics scale. An immediate consequence of the above Lagrangian is that the SM Higgs boson mediates tree level flavor changing neutral currents. Indeed, the lepton mass matrix is given by

$$
M_{i j}=v\left(Y_{i j}^{(0)}+Y_{i j}^{(1)} \frac{v^{2}}{M^{2}}\right),
$$

whereas the matrix of couplings of the physical Higgs boson is

$$
\mathcal{Y}_{i j}=Y_{i j}^{(0)}+3 Y_{i j}^{(1)} \frac{v^{2}}{M^{2}}
$$

where we have used the convention $H^{0}=v+h / \sqrt{2}$. Clearly, these matrices are in general misaligned in flavor space and the Higgs couplings in the mass eigenstate basis (cf. Eq. 2) can

\footnotetext{
${ }^{2}$ In 2 Higgs doublet models, Barr-Zee contributions often dominate at low $\tan \beta$ [17.
} 
change flavor. The latter are given by

$$
y=U_{L}^{\dagger} \mathcal{Y} U_{R}
$$

where the unitary matrices $U_{L}, U_{R}$ diagonalize the lepton mass matrix, $U_{L}^{\dagger} M U_{R}=\operatorname{diag}\left(m_{e}, m_{\mu}, m_{\tau}\right)$.

To understand the constraints on the $\operatorname{dim}-6$ operators, we vary the proportion between $Y_{i j}^{(0)}$ and $Y_{i j}^{(1)} v^{2} / M^{2}$ and scan over different $U_{L}, U_{R}$. We allow for large angle rotations, as hinted by the neutrino sector, while being agnostic about the neutrino mass matrix which presumably is provided by the seesaw mechanism. The resulting $y_{i j}$ are then subject to the bounds described in the previous section.

For the scan, we use the following procedure. The Yukawa textures are generated through

$$
Y=U_{L} \frac{1}{v} \operatorname{diag}\left(m_{e}, m_{\mu}, m_{\tau}\right) U_{R}^{\dagger}
$$

by scanning over $U_{L}, U_{R}$. Modulo phase redefinitions of the lepton fields, $U_{L, R}$ can be chosen as

$$
U_{L}=V_{L}, \quad U_{R}=V_{R} \Theta
$$

with unitary $V_{L, R}$ parametrized by three mixing angles and a single phase as in Refs. [12, 18], and $\Theta$ being a diagonal phase matrix,

$$
\Theta=\operatorname{diag}\left(e^{i \phi_{1}}, e^{i \phi_{2}}, e^{i \phi_{3}}\right)
$$

subject to the constraint $\phi_{1}+\phi_{2}+\phi_{3}=0$. Note that there are 4 reparametrization-invariant phases in the lepton mass matrix since 5 out of 9 original phases can be eliminated by the phase transformations of the left-handed and right-handed leptons.

Having generated $Y_{i j}$ (setting $H \rightarrow v$ ), we split it into two pieces according to Eq. 13 . Clearly, if $Y_{i j}^{(0)}$ dominates, $M_{i j}$ and $\mathcal{Y}_{i j}$ are almost aligned, and the lepton FCNC are suppressed. The latter therefore set a bound on the allowed proportion of $Y_{i j}^{(1)} v^{2} / M^{2}$ in the lepton mass matrix. We scan over the following parameters:

- 6 angles of $V_{L}, V_{R}$

- 2 phases of $V_{L}, V_{R}$ and 2 phases of $\Theta$

- 9 complex parameters $Y_{i j}^{(0)}$

Note that, given $Y_{i j}$ and $Y_{i j}^{(0)}$, the remaining piece $Y_{i j}^{(1)} v^{2} / M^{2}$ is determined by Eq. 13 . In what follows we present our results for two cases: $Y_{i j}^{(0)}$ is varied first in the range $[0.5,1] \times Y_{i j}$ and 

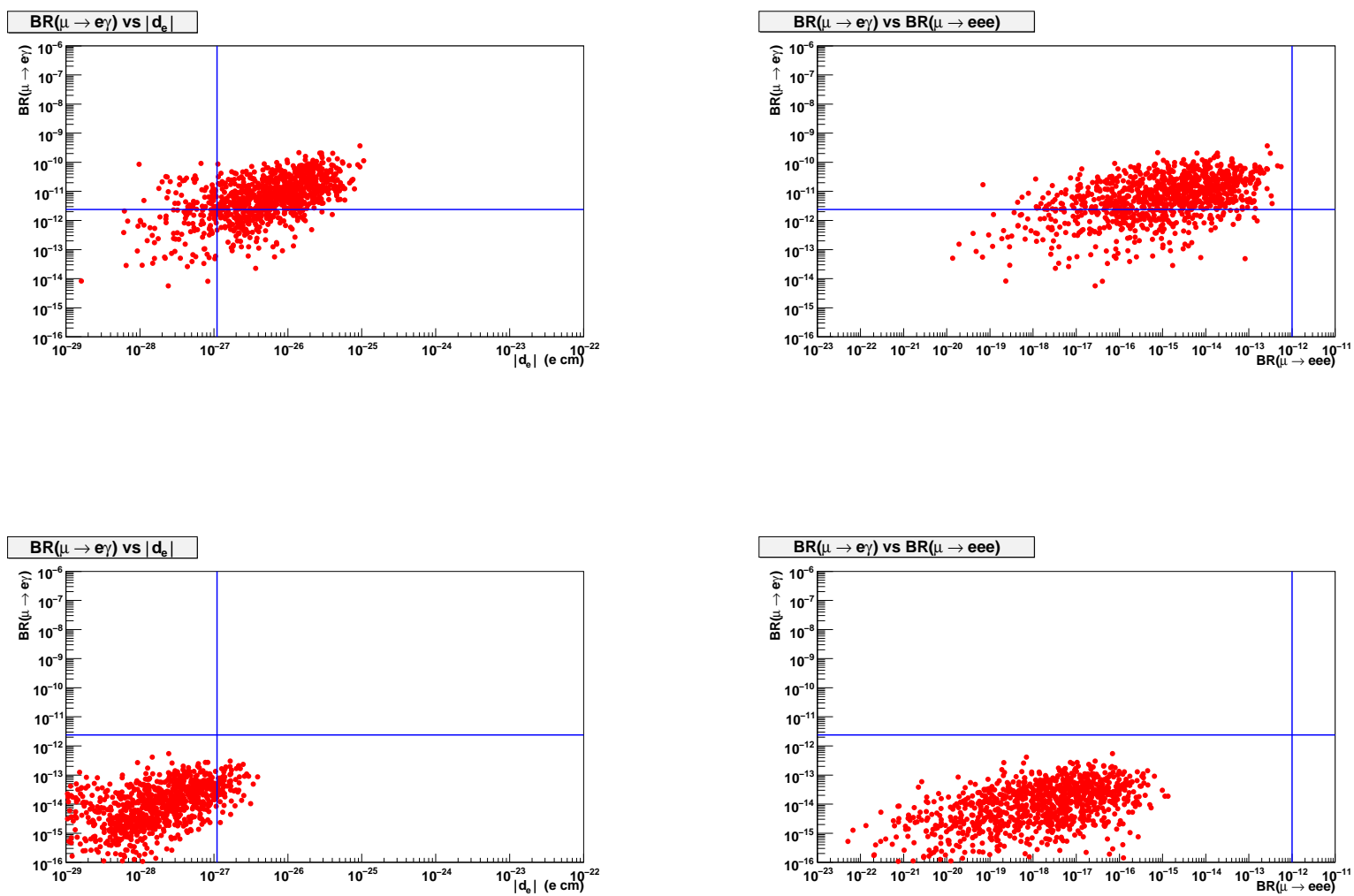

Figure 3: $\mathrm{BR}(\mu \rightarrow e \gamma)$ vs $\left|d_{e}\right|$ (left) and $\operatorname{BR}(\mu \rightarrow e \gamma)$ vs $\mathrm{BR}(\mu \rightarrow$ eee) (right) for arbitrary rotation angles and $m_{h}=200 \mathrm{GeV}$. Dimension-6 operators contribute up to $50 \%$ (top) and $10 \%$ (bottom) to $Y_{i j}$. The experimental limits are given by the straight lines.

then in the range $[0.9,1] \times Y_{i j}{ }^{3}$ This restricts the relative contribution of the dim- 6 term to no more than $50 \%$ and $10 \%$, respectively.

Our results are presented in Fig. 3. We see that if the dim-6 operators are allowed to contribute as much as $50 \%$ to $Y_{i j}, \mathrm{BR}(\mu \rightarrow e \gamma)$ and $d_{e}$ are overproduced by up to 2 orders of magnitude. On the other hand, if this contribution is below 10\%, most points are allowed for $m_{h}=200 \mathrm{GeV}$. Thus, allowing for arbitrary rotation angles, we find an empirical constraint

$$
\left|Y_{i j}^{(1)} \frac{v^{2}}{M^{2}}\right|<0.1\left|Y_{i j}\right| \times \frac{200 \mathrm{GeV}}{m_{h}} .
$$

This can be reinterpreted in terms of the bounds on the new physics scale $M$. For the two

\footnotetext{
${ }^{3}$ This range applies separately to the real and imaginary parts of $Y_{i j}^{(0)}$.
} 
limiting cases of similar $Y_{i j}^{(1)}$ and $Y_{i j}$, and order one $Y_{i j}^{(1)}$, we get

$$
\begin{gathered}
Y_{i j}^{(1)} \sim Y_{i j} \Rightarrow M>500 \mathrm{GeV} \times \frac{200 \mathrm{GeV}}{m_{h}}, \\
Y_{i j}^{(1)} \sim 1 \Rightarrow M>200 \mathrm{TeV} \times \frac{200 \mathrm{GeV}}{m_{h}} .
\end{gathered}
$$

In the latter case, we used the most restrictive Yukawa couplings $\mathcal{O}\left(10^{-5}\right)$ involving the electron. Let us note that we find observables other than $\mathrm{BR}(\mu \rightarrow e \gamma)$ and $d_{e}$ far less constraining and also confirm numerically that the diagrams with muons/electrons in the loop are unimportant. The latter statement is easy to understand. For the $\tau$ contribution, the relevant Higgs vertices involve the $\tau$ mass times a mixing parameter, e.g.

$$
y_{13} \sim \epsilon_{13} \frac{m_{\tau}}{v}
$$

and, in addition, the mass insertion in the loop is $m_{\tau}$. This enhances the $\tau$ loop by orders of magnitude. The couplings involving only the first two generations are typically bounded by $m_{\mu} / v \sim 10^{-3}$, unless there is a very strong mixing with the $\tau$. To account for the muon loop contribution to $\operatorname{BR}(\mu \rightarrow e \gamma)$ and $d_{e}$, one can simply rescale the bounds of Table 1 by $\sqrt{m_{\tau} / m_{\mu}}$ and replace index 3 by 2 . Keeping in mind that there are mixing angles appearing at the vertices so that the actual couplings are smaller than $10^{-3}$, one finds that these constraints are satisfied automatically.

As the next step, we study constraints on the rotation angles parametrizing $U_{L, R}$, while allowing for arbitrary values of the four phases therein as well as a wide range of proportions between $Y_{i j}^{(0)}$ and $Y_{i j}^{(1)} v^{2} / M^{2}$ in Eq. 13 . Specifically, we choose $Y_{i j}^{(0)}$ in the range $\pm 0.9 \times Y_{i j}$. The results are presented in Fig. 4, where we vary all the angles (in $U_{L}$ and $U_{R}$ ) in the same range. If one allows for angles as large as 0.1 , both $\mathrm{BR}(\mu \rightarrow e \gamma)$ and $d_{e}$ are overproduced, while the angles of order 0.03 are typically consistent with the constraints for $m_{h}=200 \mathrm{GeV}$. More precisely, $\operatorname{BR}(\mu \rightarrow e \gamma)$ and $d_{e}$ are most sensitive to the 1-3 and 2-3 mixing angles $\theta_{13}, \theta_{23}$ in terms of the standard parametrization of unitary matrices [18]. We then find

$$
\theta_{13}, \theta_{23}<3 \times 10^{-2} \times \frac{m_{h}}{200 \mathrm{GeV}},
$$

while $\theta_{12}$ is allowed to be as large as $\mathcal{O}(1)$. We note that $d_{e}$ is somewhat more restrictive than $\mathrm{BR}(\mu \rightarrow e \gamma)$ and requires $\theta_{13}, \theta_{23}<10^{-2}$ for $m_{h}=200 \mathrm{GeV}$. 

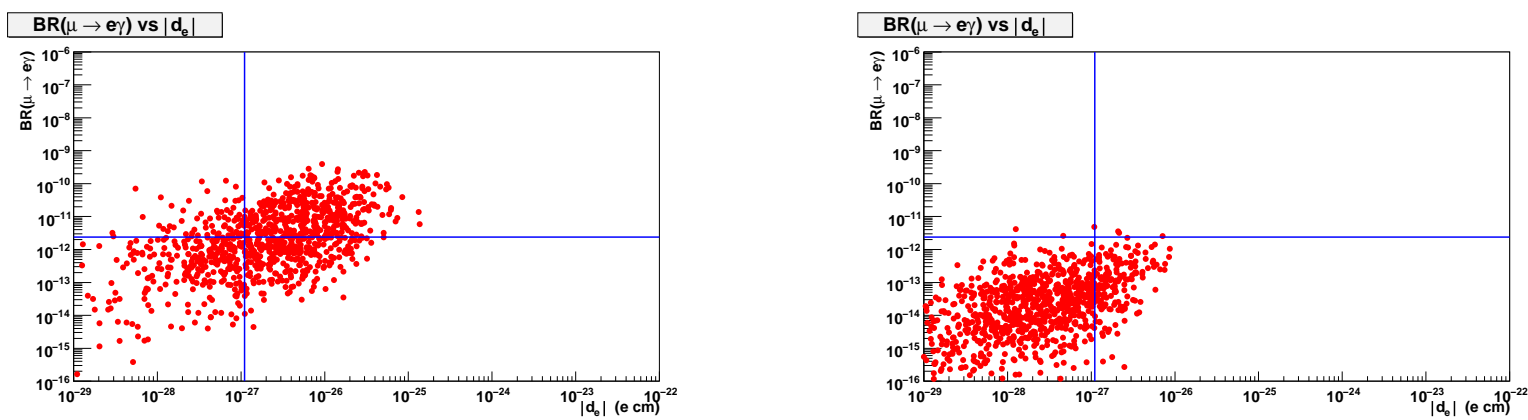

Figure 4: $\operatorname{BR}(\mu \rightarrow e \gamma)$ vs $\left|d_{e}\right|$ for small rotation angles $\theta$. Left: $\theta<0.1$, right: $\theta<0.03$. Here $m_{h}=200 \mathrm{GeV}$. The experimental limits are given by the straight lines.

\subsubsection{2-loop constraints}

The 2-loop contributions are very sensitive to the couplings in the (12)-block. In particular, if one assumed a "natural" value of $y_{12}$ to be of order $\sqrt{m_{e} m_{\mu}} / v, \operatorname{BR}(\mu \rightarrow e \gamma)$ would be overproduced by orders of magnitude, as seen from Table 2. The resulting constraints are very strong, as our numerical study demonstrates in Fig. 5. An analog of Eq. 20 in the (12)-sector reads

$$
\left|Y_{i j}^{(1)} \frac{v^{2}}{M^{2}}\right|<10^{-3}\left|Y_{i j}\right|
$$

for $m_{h}=200 \mathrm{GeV}$. The Higgs mass dependence is weaker than linear and the bound gets tighter by a factor 1.5 for $m_{h}=100 \mathrm{GeV}$. Interpreting this constraint as is done in Eq. 21, we get

$$
\begin{gathered}
Y_{i j}^{(1)} \sim Y_{i j} \Rightarrow M>5 \mathrm{TeV}, \\
Y_{i j}^{(1)} \sim 1 \Rightarrow M>2000 \mathrm{TeV} .
\end{gathered}
$$

Allowing for arbitrary proportions between $\operatorname{dim}-4$ and $\operatorname{dim}-6$ operators, we find the following constraints on the rotation angles

$$
\theta_{12}<10^{-3}, \theta_{13,23}<10^{-2}
$$

Note that $\operatorname{BR}(\mu \rightarrow e \gamma)$ is also sensitive to $\theta_{13,23}$, although not as much as to $\theta_{12}$. Unlike in the 1-loop case, a diagonal lepton Yukawa matrix does not solve all the problems: $d_{e}$ is sensitive to the phase of the flavor-diagonal coupling $y_{11}$ which does not vanish in the limit of zero rotation 

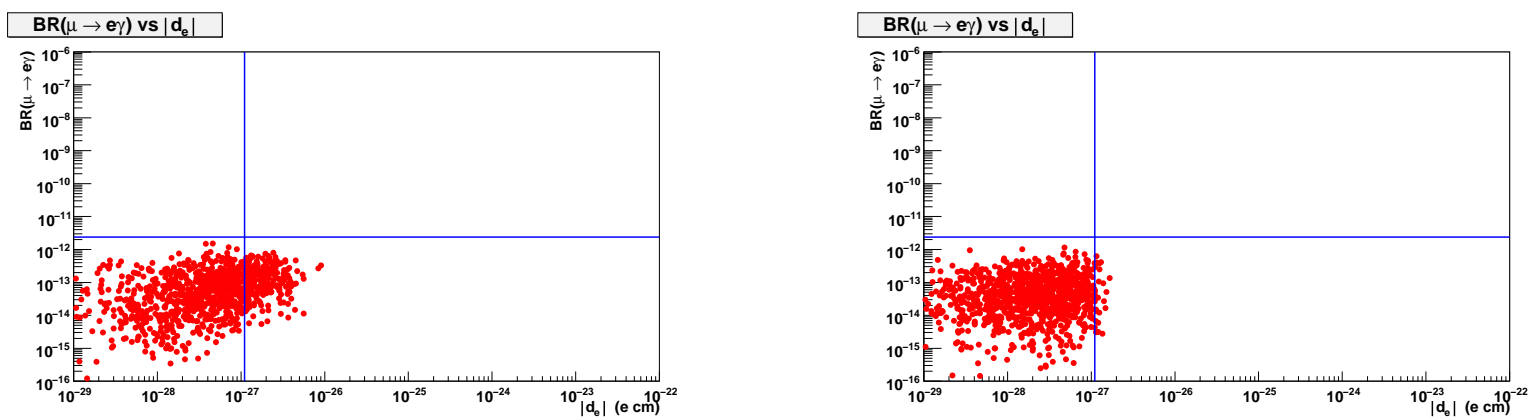

Figure 5: $\operatorname{BR}(\mu \rightarrow e \gamma)$ vs $\left|d_{e}\right|$ at 2 loops. Left: the dim-6 contribution is limited to $0.1 \%$, while the rotation angles are arbitrary. Right: the rotation angles are bounded by $\theta_{12}<10^{-3}, \theta_{13,23}<$ $10^{-2}$ and the CP phases $\phi<0.1$, while the balance between dim- 4 and dim- 6 contributions is arbitrary. Here $m_{h}=200 \mathrm{GeV}$. The experimental limits are given by the straight lines.

angles. We thus have a separate constraint on the CP phase of $y_{11}$ :

$$
\phi<10^{-1}
$$

\subsection{Higher dimensional operators and the mass hierarchy}

Let us now explore the possibility that the lepton mass hierarchy is entirely due to higher dimensional operators [1, 2]. The Yukawa couplings are expanded as

$$
Y_{i j}(H)=\sum_{n_{i j}=0}^{\infty} \kappa_{i j}^{\left(n_{i j}\right)}\left(\frac{H^{\dagger} H}{M^{2}}\right)^{n_{i j}}
$$

with order one $\kappa_{i j}^{\left(n_{i j}\right)}$ and $M$ being a new physics scale. In most interesting cases which address the flavor problem, the coefficients $\kappa_{i j}^{\left(n_{i j}\right)}$ vanish up to a certain order $n_{i j}$. This can happen due to some symmetry (e.g. Froggatt-Nielsen type [19]) of the UV completion of our effective theory, which may not be apparent at low energies. In this case, the mass hierarchy is generated by

$$
\epsilon=\frac{v^{2}}{M^{2}} \ll 1
$$

and the Yukawa textures take the form

$$
Y_{i j}=c_{i j} \epsilon^{n_{i j}}
$$


with order one $c_{i j}$. Analogous textures in the quark sector were considered in [2]. It is interesting that the invariant measure of $\mathrm{CP}$ violation increases by many orders of magnitude compared to that in the Standard Model, which can be relevant to baryogenesis [20].

Various lepton textures of the above type can be generated as follows. The Yukawa matrix is represented in terms of the eigenvalues and the rotation matrices $U_{L, R}$ as in Eq. 17 . Scanning over $U_{L, R}$ then produces viable lepton textures. Choosing for definitness $\epsilon=1 / 60$ as motivated by the top-bottom quark mass hierarchy [2], one determines the exponents $n_{i j}$ via

$$
n_{i j}=\operatorname{round}\left(\log _{\epsilon}\left|Y_{i j}\right|\right)
$$

which also fixes the $\mathcal{O}(1)$ coefficients $c_{i j}$. Moving to the mass eigenstate basis, we obtain the lepton couplings of the physical Higgs (2),

$$
y_{i j}=\left(U_{L}\right)_{k i}^{*}\left(2 n_{k l}+1\right) Y_{k l}\left(U_{R}\right)_{l j}
$$

Note that, in this basis, $y_{i j}$ are defined up to a phase (see [20] for details). Indeed, one can multiply the left- and right-handed leptons by diagonal phase matrices such that the mass terms remain the same. However, observables are invariant under this reparametrization since they involve combinations like $y_{3 i} y_{i 3}$ or absolute values of the couplings.

For concreteness, let us restrict ourselves to Yukawa textures of the following type

$$
Y \sim\left(\begin{array}{ccc}
\epsilon^{3} & \epsilon^{l} & \epsilon^{m} \\
\epsilon^{l} & \epsilon^{2} & \epsilon^{n} \\
\epsilon^{m} & \epsilon^{n} & \epsilon^{1}
\end{array}\right) .
$$

Here we have chosen the diagonal entries such that they reproduce (roughly) the $m_{e}: m_{\mu}: m_{\tau}$ hierarchy. The integers $l, m, n$ are subject to the LFV and CP constraints. For large $l, m, n$ the Yukawa matrix is approximately diagonal and the constraints are satisfied. Numerically, we find the following approximate bound:

$$
l \geq 4, \quad m \geq 2, \quad n \geq 2 .
$$

For most choices of order one coefficients in the texture, the constraints are satisfied in this case. Note that the bound on $l$ is the strongest one due to the Barr-Zee contributions to $\mu \rightarrow e \gamma$. This entails, in particular, that the lepton analog of the down quark texture studied in Ref. [2] is strongly disfavored.

Our numerical results for representative textures are shown in Fig. 6. We take $l=4$, $m=n=2$ for panel (a) and $l=m=2, n=1$ for panel (b). In this figure, the light 


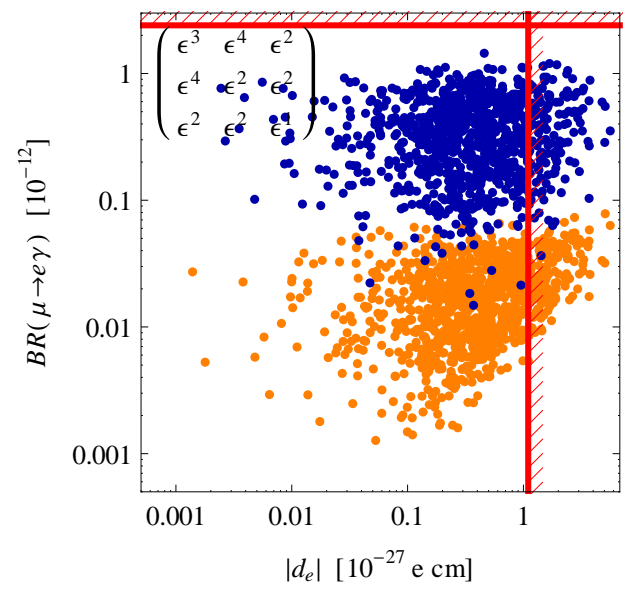

(a)

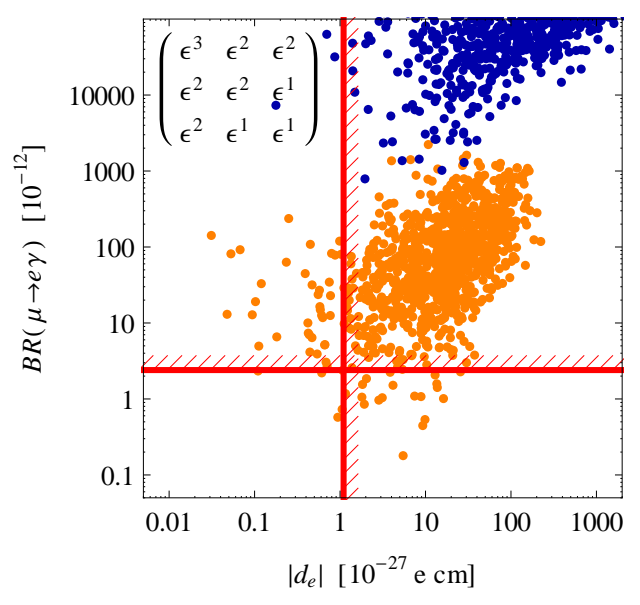

(b)

Figure 6: $\operatorname{BR}(\mu \rightarrow e \gamma)$ vs $\left|d_{e}\right|$ for representative Yukawa textures, with $m_{h}=200 \mathrm{GeV}$. Light (orange) and dark (blue) dots correspond to one loop and one + two loop contributions, respectively. The experimental limits are given by the straight lines.

(orange) and dark (blue) dots correspond to one loop and one loop plus two loop contributions, respectively. For case (a) most points satisfy the constraints, whereas in case (b) the bounds are grossly violated. We find that $\operatorname{BR}(\mu \rightarrow e \gamma)$ is dominated by the Barr-Zee contributions, whereas $d_{e}$ receives comparable contributions at 1 and 2 loops. As expected, significant departures from the diagonal Yukawa form, especially in the (12)-block, are not allowed.

Finally, let us note that although the constraints are strong, the remarkable sensitivity of $\mu \rightarrow e \gamma$ and $d_{e}$ also implies good prospects for detection of Higgs-induced lepton flavor and CP violation in current and future experiments.

\section{Conclusion}

In this work, we have derived model-independent bounds on the lepton flavor violating couplings of the SM Higgs boson. Such flavor violation appears when higher dimensional operators contribute to the lepton masses. Scanning over various textures shows that these contributions should be limited to about $0.1 \%$ in the (12)-sector and no more than $10 \%$ in the (13) and (23)-sectors, otherwise $\operatorname{BR}(\mu \rightarrow e \gamma)$ and $d_{e}$ are overproduced. Alternatively, if one allows for large contributions of the higher order operators, the Yukawa matrix must be diagonalizable by a small-angle rotation with $\theta_{12} \sim \mathcal{O}\left(10^{-3}\right)$ and $\theta_{13}, \theta_{23} \sim \mathcal{O}\left(10^{-2}\right)$. In addition, the electron EDM sets a constraint on the relevant CP phase $\phi<\mathcal{O}\left(10^{-1}\right)$. 
Further, we have studied the possibility that the lepton mass hierarchy is created entirely by non-renormalizable operators. Also in this case the LFV effects are significant. The preferred textures have small intergenerational mixing, especially in the (12)-sector, as required by the Barr-Zee contributions to $\mu \rightarrow e \gamma$. As a result, the lepton analog of the down quark texture studied in [2] is strongly disfavored.

On the other hand, the remarkable sensitivity of LFV and CP violating observables to Higgs-induced effects implies good experimental prospects for detection of $\mu \rightarrow e \gamma$ and $d_{e}$.

\section{Acknowledgments}

We are grateful to S. Davidson for useful comments. The work of A.G. is supported in part by the Landes-Exzellenzinitiative Hamburg. J.P. is supported in part by German Research Foundation DFG through Grant No. STO876/2-1.

\section{References}

[1] K. S. Babu and S. Nandi, Phys. Rev. D 62, 033002 (2000).

[2] G. F. Giudice and O. Lebedev, Phys. Lett. B 665, 79 (2008).

[3] R. Lafaye, T. Plehn, M. Rauch, D. Zerwas and M. Duhrssen, JHEP 0908, 009 (2009).

[4] J. D. Bjorken, S. Weinberg, Phys. Rev. Lett. 38, 622 (1977).

[5] B. McWilliams, L. -F. Li, Nucl. Phys. B179, 62 (1981).

[6] O. U. Shanker, Nucl. Phys. B206, 253 (1982).

[7] J. L. Diaz-Cruz, J. J. Toscano, Phys. Rev. D62, 116005 (2000). See also an analogous study in the quark sector, F. del Aguila, M. Perez-Victoria, J. Santiago, Phys. Lett. B492, 98-106 (2000).

[8] T. Han, D. Marfatia, Phys. Rev. Lett. 86, 1442-1445 (2001).

[9] J. I. Aranda, F. Ramirez-Zavaleta, J. J. Toscano, E. S. Tututi, Phys. Rev. D78, 017302 (2008).

[10] F. Bonnet, D. Hernandez, T. Ota, W. Winter, JHEP 0910, 076 (2009); M. B. Krauss, T. Ota, W. Porod, W. Winter, arXiv:1109.4636 [hep-ph]]. 
[11] A. J. Buras, M. V. Carlucci, S. Gori, G. Isidori, JHEP 1010, 009 (2010).

[12] K. Nakamura et al. (Particle Data Group), J. Phys. G 37, 075021 (2010) and 2011 partial update for the 2012 edition.

[13] MEG collaboration, arXiv:1107.5547 [hep-ex].

[14] J. Girrbach, S. Mertens, U. Nierste and S. Wiesenfeldt, JHEP 1005 (2010) 026.

[15] S. M. Barr, A. Zee, Phys. Rev. Lett. 65, 21-24 (1990).

[16] D. Chang, W. S. Hou, W. -Y. Keung, Phys. Rev. D48, 217-224 (1993).

[17] S. Davidson and G. J. Grenier, Phys. Rev. D 81, 095016 (2010).

[18] L. L. Chau and W. Y. Keung, Phys. Rev. Lett. 53 (1984) 1802.

[19] C. D. Froggatt and H. B. Nielsen, Nucl. Phys. B 147, 277 (1979).

[20] O. Lebedev, Phys. Lett. B 697, 58 (2011). 